\title{
Investigation of Performance Gains on a Sailplane with Morphing Wing Trailing Edge
}

\author{
Martynas LENDRAITIS \\ Kaunas University of Technology, Studentu st. 56, 51424, Kaunas, Lithuania, E-mail: martynas.lendraitis@ktu.lt

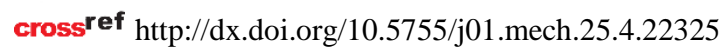

\section{Introduction}

Competition sailplanes are the most aerodynamically optimized aircraft. The requirement to fly long distances in both high and low speeds requires sailplanes to have high lift to drag ratios through the whole flight envelope. With recent development in aerodynamics, when most of the flow around the sailplane wing is laminar, there is not much improvements possible which could substantially increase the performance even more. Researchers are now seeking solutions to overcome these limits. One solution is to use boundary layer control devices which would enable laminar flow to extend even further and theoretically allow reaching a lift to drag ratio of as much as 100 for an open class sailplane [1]. But this requires using special pumps, which require power, neglecting the philosophy of what a pure sailplane is.

Another solution, which is being investigated in other aviation fields, is the use of morphing structures. Such structures could allow designing a wing that would be optimal at various flight conditions. Full morphing wings are being tested in wind tunnels with promising results, but are still far from full implementation [2, 3]. A much easier approach is using only small wing sections for morphing. Most researchers are now investigating how to replace conventional flaps with morphing flaps for better efficiency. Their approaches suggest that even with a small portion of morphing wing a fuel consumption reduction of more than $5 \%$ is possible on conventional aircraft [4]. Some of the structural designs are already applied on actual flying aircraft showing the maturation of some technologies [5]. These approaches are highly applicable to modern sailplanes with possibility of retrofitting current sailplanes with morphing flaps.

Most sailplanes use conventional flaps for flight performance alteration. Small flap deflections allow to fly faster or slower with minimum energy loses. Most of the best sailplane airfoils are designed specifically for the use of flaps, and researchers are taking vast amount of effort on optimizing such cases [6]. Unfortunately, by designing an airfoil for the use with a flap, some compromises must be made. In some cases, a sailplane designed with a wing without flaps (standard class), could have an advantage at some speed compared to a sailplane with flapped wings. By the use of morphing flap, all of the flap settings could be made optimal.

Yet, there is no data on any investigation on a trailing edge morphing flap for a modern sailplane. A simple case is investigated in this work.

\section{Optimization methods}

The investigation of possible performance improvement on a sailplane must start form an airfoil investigation and optimization. The selection of such airfoil might contribute largely to the performance gains achieved, thus the selected airfoil should be used on some kind of existing sailplane. It is difficult to find public data on modern sailplane airfoils, because most of them are a property of particular sailplane manufacturers. Thus, the author was limited to the publicly available airfoils. Therefore, an older airfoil HQ-17 (Fig. 1) was chosen. This airfoil is used in several sailplanes, including ASW22 [7], which is still considered competitive by today's standards.

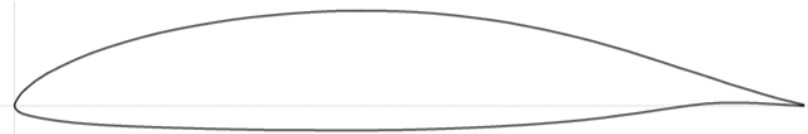

Fig. 1 Airfoil HQ-17

The optimization was carried out on the trailing edge section, starting from $80 \%$ from the leading edge. The trailing edge was fixed at locations corresponding to +12 and -4 flap deflection positions. These settings were chosen to investigate the performance when flying at low and high speeds.

For optimization, a special MATLAB script was written. Aerodynamic optimization is carried out on an airfoil section by changing the surface shape on specified location of the trailing edge. The trailing edge surface is replaced with a B-spline curve. The curve is defined as a linear combination of control points $p_{i}$ and the B-spline basis function $N_{i, k}(t)$ given by [8]:

$$
r(t)=\sum_{t=0}^{n} p_{i} N_{i, k}(t), \quad n \geq k-1, \quad t \in\left[t_{k-1}, t_{n+1}\right]
$$

where: $t$ is nodes.

The shape is altered by changing the location of the control points which are controlled by a genetic algorithm from MATLAB software [9]. The algorithm objective function is specified as the minimization function of drag at specified lift coefficients. When the lift is fixed, alteration of drag changes the lift to drag ratio at the specific point. This is done, because the lift coefficient at a given flight weight and horizontal flight speed is fixed. Therefore, it is reasonable to minimize the drag for a specific lift coefficient, thus shifting the drag polar to the left.

It is well to note, that in order to get reasonable results, multiple optimization points must be chosen. The number of optimization points should be reasonably high 
and well spread across the polar. If the number is too small, or the points are too close, they could form an uneven polar, where desired results are reached only on those locations, resulting in poor performance in other areas [10]. The described objective function can be written:

$$
\operatorname{minimize} \quad c_{\text {d.average }}=\frac{\sum_{i=1}^{n} c_{d . i}}{n} ;
$$

where: $n$ is the number of optimization points.

It's a good practice to add an additional weighting coefficient to all $c_{d}$ values in order to achieve a more consistent drag polar. But in this case, it wasn't used because of satisfactory outcomes with the selected objective function.

Software XFOIL [11] is used to calculate the airfoil section performance for the objective function. XFOIL has proven to be a very fast, robust and accurate airfoil calculation tool even when compared with more modern calculation approaches such as CFD [12, 13]. XFOIL is orders of magnitude faster, thus useful for optimization. Some limitations still exist, but it's well managed by using certain methods. Some researchers suggest modifying $N_{\text {crit }}$ values for upper and lower wing surfaces in XFOIL could yield a significant improvement in calculation accuracy when dealing with sailplane airfoils [6].

During the optimization, population of multiple randomly generated airfoils are defined first. After evaluating performance with the objective function, best designs are chosen. These designs, which are called "elite individuals" are carried over to the next generation. Some airfoils are merged to form new ones; this procedure is called recombination. After a certain number of generations, the solution starts to converge and within set boundaries, best design is found.

\section{Flap optimization}

Using the described algorithm, optimization is carried out on the trailing edge of the HQ-17 airfoil with a fixed flap deflection. The modification of the surface starts at 0,8 of the chord, where the conventional flap hinge might be placed. After evaluating usual flap settings in sailplanes, $-4^{\circ}$ and $+12^{\circ}$ flap deflection angles were chosen for optimization. These flap deflection angles are commonly used in modern sailplanes. Usually even bigger positive deflections are used when thermaling, but it was decided to investigate an intermediate case.

$-4^{\circ}$ flap deflection is used for high speed flying from one thermal to the other and $+12^{\circ}$ is used for slow speed, when flying in thermals. For optimizing $-4^{\circ}$ flap, a Reynolds number of $2 \mathrm{M}$ was selected. For $+12^{\circ}$ flap deflection case, Reynolds number of $1 \mathrm{M}$ was selected. Only two optimization scenarios are evaluated in this research.

Optimizations were carried out at specific lift coefficient $c_{l}$ range which allows reaching the intended improvement. At -4 flap deflection, the objective function was evaluated for low $c_{l}$ values: from 0.2 to 0.6 with steps of 0.05 . On $+12^{\circ}$ flap deflection, $c_{l}$ optimization range was set from 1 to 1.4. This $c_{l}$ range is particularly useful for slow speed flying. For every flap optimization case, 9 optimization point were used.
To find the optimal solution, a genetic algorithm must have a sufficiently large population size. However, a population that is too large usually leads to a higher calculation time with little improvement. Considering available computer resources, the population size was set to 50 individuals. Recombination was set to 0.8 and the elite individual count to 6 .

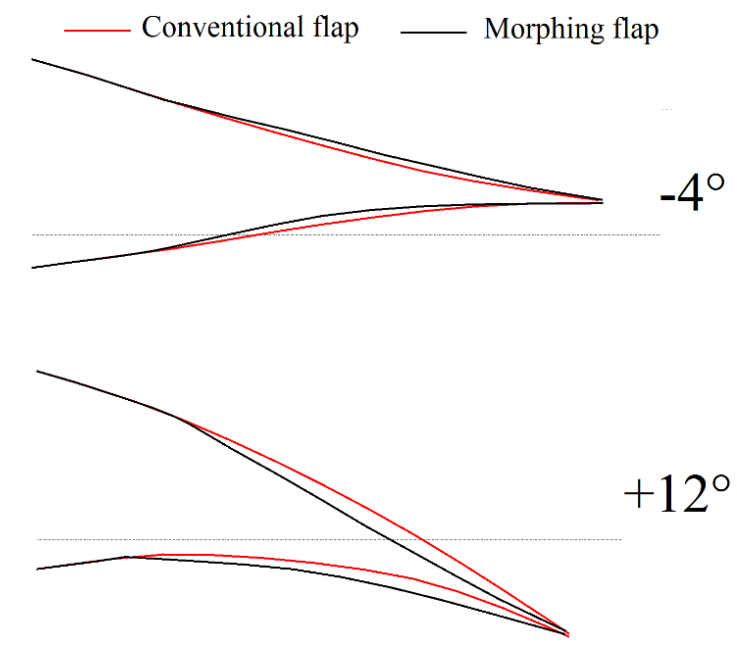

Fig. 2 Conventional and morphing flap geometry

Optimized geometry is shown in Fig.2. The shape difference between conventional and morphing flaps is clearly seen. As expected, the morphing flap generally has a smoother curve. The drag polars for these flaps are given in Figs. 3 and 4 at Reynolds number of $R e=1 \cdot 10^{6} / \sqrt{ } c_{1}$. The graphs are shown as Type 2 drag polars, which corresponds to a sailplane in level flight undergoing trim speed changes, adjusting the $R e$ number for every lift coefficient.

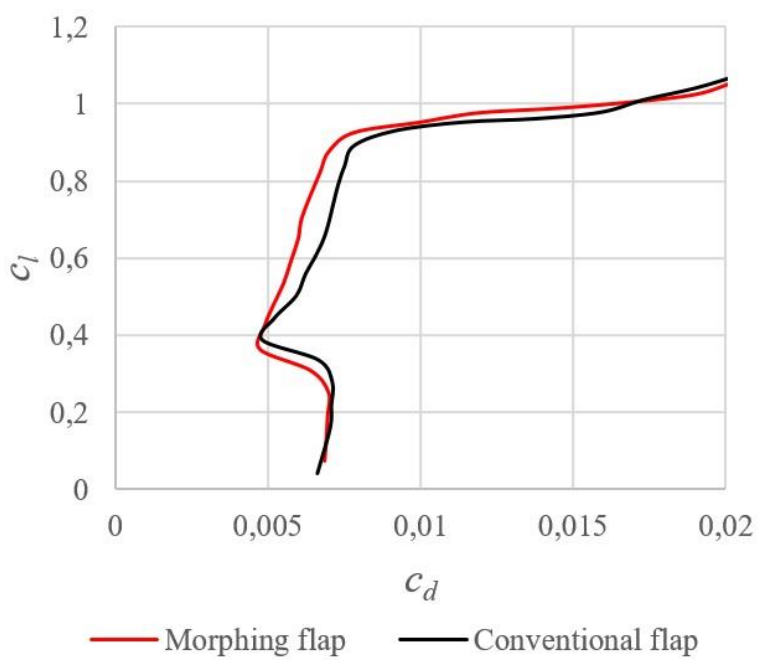

Fig. 3 Drag polar of airfoil HQ-17 with conventional and morphing flaps at deflection angle of $-4^{\circ} . R e=1 \cdot 10^{6}$ $/ \sqrt{ } c_{l}$

Optimization at -4 degrees flap deflection (Fig. 3) shows a more consistent performance than the conventional flap. It is seen that the drag improvement reaches up to $8 \cdot 10^{-4}(12 \%)$ at $c_{l}=0.6$. At $c_{l}=0.2$ and 0.4 not much improvement is seen. However, between these lift coefficients a drag reduction of up to $1 \cdot 10^{-3}$ can be seen. The minimum drag is reached at a lower lift coefficient, suggesting superior highspeed performance. 


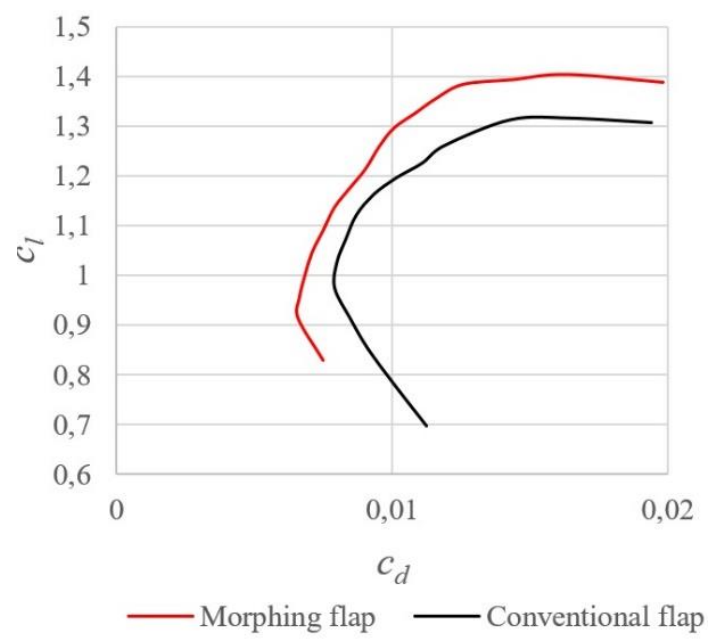

Fig. 4 Drag polar of airfoil HQ-17 with conventional and morphing flap at deflection angle of $12^{\circ} . R e=1 \cdot 10^{6}$ $/ \sqrt{ } c_{l}$

Fig. 4 shows the flap with $+12^{\circ}$ deflection. A higher maximum lift coefficient and lower drag than that of the conventional flap is seen. As intended by the optimizer, the maximum lift coefficient reaches 1.4. Drag is reduced by about $1.2 \cdot 10^{-3}(15 \%)$ at $c_{l}=1$ and $5 \cdot 10^{-3}(36 \%)$ at $c_{l}=1.3$. The performance is significantly better, especially at higher angles of attack, as was the intention of the optimization.

\section{Speed polar estimation}

To evaluate what performance benefits such airfoil modification could yield on an actual sailplane, a hypothetical sailplane was designed. Non-linear LLT (Lift line theory) from the program XFLR5 [14] is chosen for the performance gain estimation. It provides more accurate solution than linear LLT. In contrast to traditional LLT, non-linear LLT considers viscous effects on the wing by interpolating the non-linear section characteristics at multiple wing sections, which are calculated by XFOIL. This method is suitable for performing an analysis on medium to high aspect ratio wings with no dihedral and low sweep angle $[14,15]$.

As the wing planform example, a modern high-performance sailplane LAK-17B with an 18m wing span was chosen. The planform data is taken from the sailplane maintenance manual [16]. The wing area was set to $10.3 \mathrm{~m}^{2}$ with a $0.606 \mathrm{~m}$ mean chord and an aspect ratio of 31 .

The wing is modeled keeping a constant airfoil through the wingspan. The flap is modeled almost through the whole wing, leaving only small sections at the root and the tip without the flap (similarly as in LAK-17B).

The junctions between the flap and the wing are modelled in a different manner. The conventional configuration has a gap (Fig. 5 a), which allows the flow to pass from lower surface and form a vortex which increases overall drag. The morphing wing has a smooth transition with no gap in between (Fig. 5 b). This small adjustment has a significant impact on the sailplane performance. However, the actual impact from the vortex might be bigger than estimated by the LLT non-linear method, because of the complex flow generated there, which LLT is not capable of simulating.

The designed wing with 1100 panels is shown in Fig. 6. The sailplane mass was designed to be $490 \mathrm{~kg}$. Because the analysis is done only on the wing, additional drag was added to simulate the full sailplane performance. Extra drag corresponding to $2.5 \mathrm{~m}^{2}$ reference area with a 0.0125 drag coefficient was added. This corresponds to the drag generated by the fuselage and the tail. With additional drag the maximum L/D was lowered to 50, which is close to the values experienced by similar sailplanes.

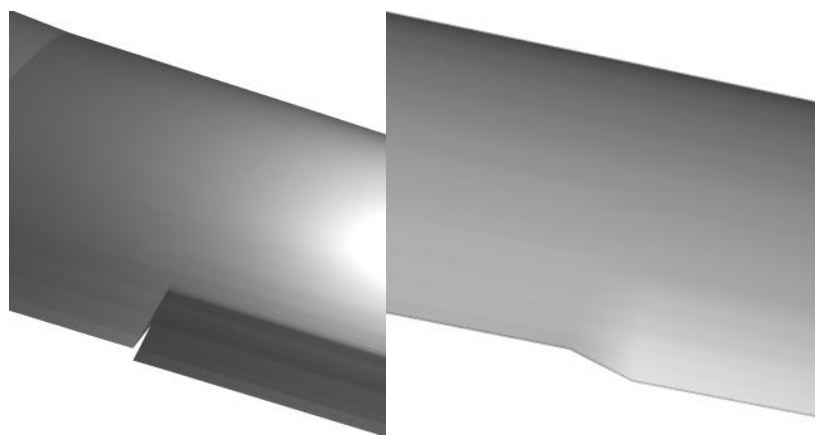

a

b

Fig. 5 Flap transition from the fixed section of the wing. A - conventional flap b - morphing flap

Fig. 6 Sailplane wing with panels

The sailplane speed polar is a common method to display sailplane performance. It shows the sailplane's flight speed dependency on its sink speed. It's important for sailplane pilots to determine the best speed to fly in different flight stages. Most of the time, the sailplane performance is determined by the maximum $L / D$ ratio, but in recent years this number became less important, because it doesn't reflect the actual performance in the full flight envelope. Most of the improvement in sailplane performance comes from the flattening of the speed polar. A shallower polar suggests more consistent performance, which is a more suitable for fast cross-country soaring flights. The performance can also be increased by extending the polar itself. The maximum speed is determined by the maximum structural load the sailplane can handle, thus it is of little interest when dealing with aerodynamic performance. But extending polar to the left means expanding the sailplane flight envelope by allowing it to fly slower.

By incorporating optimized morphing flap to hypothetical sailplane, benefits are clearly seen. Figure 7 shows the speed polar comparison between morphing and conventional flaps at -4 degree deflection. The morphing flap shows a consistent improvement on sink speed reduction of about $0.027 \mathrm{~m} / \mathrm{s}$ from 28 to $40 \mathrm{~m} / \mathrm{s}$ flight speed. This 
improvement is equivalent to about 2 units of $\mathrm{L} / \mathrm{D}$ or an improvement of about $2 \%$, which is significant. At velocities of 40 to $44 \mathrm{~m} / \mathrm{s}$ there is not much difference between the polars. This region of the speed polar is reached close to an airfoil section lift coefficient of 0.4 , where drag is similar to the conventionally flapped airfoil (as seen in Fig. 3). At an even higher speed, the descent speed is reduced by up to 0.1 $\mathrm{m} / \mathrm{s}$, equivalent to an L/D improvement of nearly 2 units or almost 5 percent.

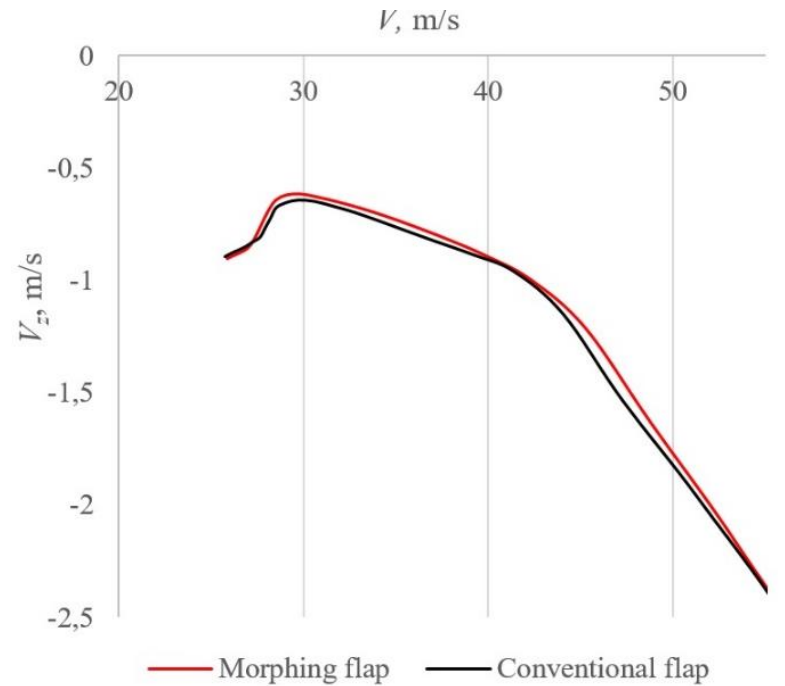

Fig. 7 Speed polar at $-4^{\circ}$ flap deflection

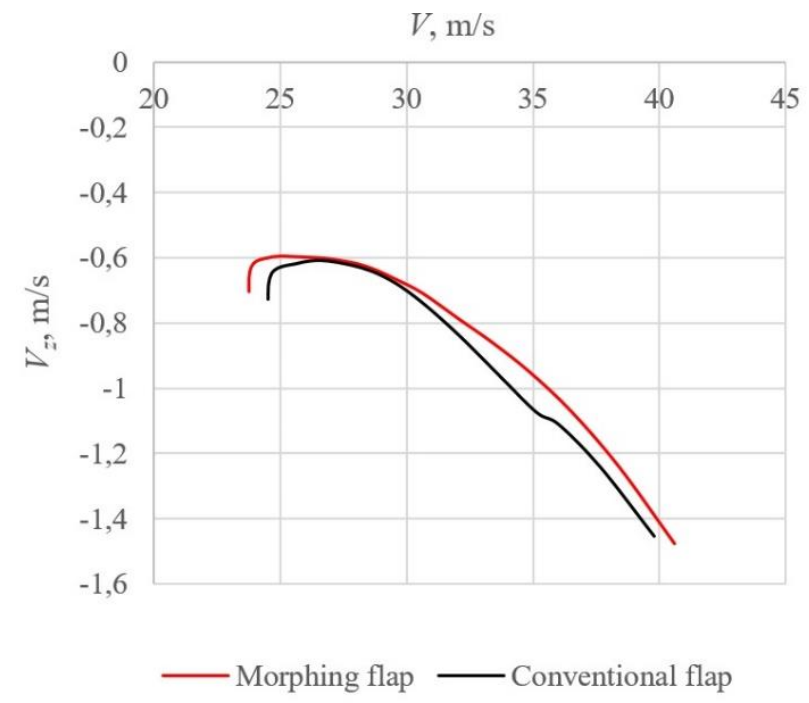

Fig. 8 Speed polar at $+12^{\circ}$ flap deflection

Fig. 8 shows the speed polar with a flap deflection of $+12^{\circ}$. A noticeable low speed improvement is seen. The minimum speed is reached at an about $2 \mathrm{~m} / \mathrm{s}$ lower flight velocity than with conventionally flapped wing. The minimum sink speed is also reduced in a similar manner. Thought the optimization wasn't intended to increase the high speed performance when the flap is extended down, the sink speed has improved significantly. However, the high speed performance improvement isn't particularly useful, because there is no purpose to fly fast with this flap setting.

\section{Discussion}

It is clear that a morphing sailplane may offer significant improvement on sailplane performance. Yet, it is still a hardly manageable task, because of the complexity that comes with the actual structure. The manufacturers must decide if this transition is feasible and if they will dedicate the effort and money to design such a wing. There is no current structure solution to design such a wing, therefore it may be expensive.

A more in depth analysis should be made on a specific sailplane. The analysis presented here investigates only a hypothetical sailplane with a constant airfoil through the span. Yet, most sailplane wings consist of multiple airfoils, designed for multiple Reynolds numbers. From this perspective, optimization on multiple airfoils at different Reynolds numbers might lead to a more significant impact to the performance.

It is well to note that optimizing the trailing edge flap for some airfoils might be difficult. Most new sailplane airfoil is already designed with a flap in mind. Therefore, the airfoils shape is already defined by the flap constrains. For some airfoils, retrofitting them with a morphing flap might offer only a small or even negligible advantage. This was experienced by the author when trying to optimize the flaps on certain airfoils. The best way to extract the possible benefits of a morphing flap is to design a new airfoil from the ground up with a morphing flap in mind. This approach might lead to even bigger benefits than the case presented in the article.

Also, one should address the possible limitations of XFOIL and nonlinear LLT calculation methods, because researchers found that they are highly accurate in some cases, but not in other.

The article doesn't address the possible improvements on the handling and turn performance. The author thinks that morphing technology might be beneficial to it as well. The optimized airfoil flaps have lower drag per change in lift coefficient. This would result in a lower energy loss when turning and might reduce adverse yaw during the turn. A higher control response also might be reached.

\section{Conclusions}

The results suggest that using morphing flaps over conventional ones might be beneficial on a sailplane. The optimized airfoil flaps show a reduction in drag of up to $12 \%$ at flap settings of $-4^{\circ}$ and up to $36 \%$ with $12^{\circ}$ flap deflection on the selected HQ-17 airfoil. A hypothetical sailplane wing was modeled, and using the optimized airfoils with flaps, compared with a conventional one. The results suggest that this change results in a lift to drag ratio increased by about $2 \%$ across the flight polar at $-4^{\circ}$ flap deflection. At $+12^{\circ}$ the lift to drag ratio increased by up to $5 \%$ and the minimum flight speed was reduced by $2 \mathrm{~m} / \mathrm{s}$ with morphing flap extended.

When designing a new sailplane, morphing technology could offer a significant improvement in performance. When all the airfoils in a sailplane are designed with morphing structures in mind, even greater improvements might be reached.

\section{References}

1. Stimson, M. 2017. Future of the Open Class, Tech. Soar 41.

2. Jenett, B. et al. 2017. Digital morphing wing: active wing shaping concept using composite lattice-bBased 
cellular structuresm, Soft Robot 4: 33-48. https://doi.org/10.1089/soro.2016.0032

3. Previtali, F.; Molinari, G.; Arrieta, A. F.; Guillaume, M.; Ermanni, P. 2016. Design and experimental characterisation of a morphing wing with enhanced corrugated skin, J. Intell. Mater. Syst. Struct. 27: 278292. https://doi.org/10.1177/1045389X15595296.

4. Burdette, D. A.; Martins, J. R. R. A. 2018. Design of a transonic wing with an adaptive morphing trailing edge via aerostructural optimization, Aerosp. Sci. Technol. 81: 192-203. https://doi.org/10.1016/j.ast.2018.08.004.

5. Kota, S.; Flick, P.; Collier, F. S. 2016. Flight testing of FlexFloil ${ }^{\mathrm{TM}}$ adaptive compliant trailing edge, in 54th AIAA Aerospace Sciences Meeting. https://doi.org/10.2514/6.2016-0036.

6. Kubrynski, K. 2012. Design of a flapped ;aminar airfoil for high performance sailplane, in 30th AIAA Applied Aerodynamics Conference. https://doi.org/10.2514/6.2012-2662.

7. Simons, M. 2004. Sailplanes 1965 - 2000. 270 p.

8. Hoschek, J.; Lasser, D. 1993. Fundamentals of computer-aided geometric design, A K Peters. 752 p.

9. Saraswat, M.; Kumar Sharma, A. 2013. Genetic Algorithm for optimization using MATLAB, Int. J. Adv. Res. Comput. Sci.

10. Drela, M. 1998. Pros and cons of airfoil optimization, in Proceedings of Frontiers of Computational Fluid Dynamics. https://doi.org/10.1142/9789812815774_0019.

11. Drela, M. 1989. XFOIL: an analysis and design system for low Reynolds number airfoils, in 1-12. https://doi.org/10.1007/978-3-642-84010-4_1.

12. Morgado, J.; Vizinho, R.; Silvestre, M. A. R.; Páscoa, J. C. 2016. XFOIL vs CFD performance predictions for high lift low Reynolds number airfoils, Aerosp. Sci. Technol. 52: 207-214. https://doi.org/10.1016/j.ast.2016.02.031.

13. Coder, J. G.; Maughmer, M. D. 2014. Comparisons of theoretical methods for predicting airfoil aerodynamic characteristics, J. Aircr.
https://doi.org/10.2514/1.C032232.

14. Deperrois, A. 2013. XFLR5 Analysis of foils and wings operating at low Reynolds numbers. 71p.

15. Pakalnis, E.; Lasauskas, E.; Stankunas, J. 2005. Convergence of lift force calculation of a tapered wing using non-linear section data, Mechanika 1(51): 61-65. https://doi.org/10.5755/j01.mech.51.1.12922.

16. Sportinè aviacija ir Ko. 2015. Maintenance Manual For The Sailplanes LAK-17B, LAK-17BT. 158p.

M. Lendraitis

\section{INVESTIGATION OF PERFORMANCE GAINS ON A SAILPLANE WITH MORPHING WING TRAILING EDGE}

S u m m a r y

Increasing the performance of a modern sailplane is challenging. Most of the known valid approaches have already been applied in practice. Morphing technology, which could allow to adapt to various flight stages is yet to be applied. An investigation of possible flight performance benefits of such technology is carried out here. Using a genetic algorithm, a morphing trailing edge flap for airfoil HQ-17 is formed for $-4^{\circ}$ and $+12^{\circ}$ flap deflections. The performance is evaluated and compared with a regular flap, which shows that for the HQ-17 airfoil, drag could be reduced by up to $36 \%$ in comparison with regular flap. To show the advantage of morphing flaps, a hypothetical sailplane wing is modeled and evaluated with morphing and conventional flaps using non-linear LLT method. Results show that incorporation of a morphing flap could extend the flight envelope and increase the $L / D$ ratio by $2-5 \%$ through the full flight speed range.

Keywords: morphing, flap, aerodynamics, optimization.

Received November 06, 2018 Accepted August 26, 2019 